\title{
Home Agent placement and assignment in WLAN with Cellular Networks
}

\author{
Selvakumar.R, \\ Senior Lecturer, \\ Department of Computer \\ Science and Engineering \\ V.M.K.V.Engineering College, \\ Salem-636 308 \\ Tamilnadu, India.
}

\author{
Mohamed Saleem.S, \\ Undergraduate Student, \\ Department of Computer \\ Science and Engineering \\ V.M.K.V.Engineering College, \\ Salem-636 308 \\ Tamilnadu, India.
}

\author{
Vipul Jain.V \\ Undergraduate Student, \\ Department of Computer \\ Science and Engineering \\ V.M.K.V.Engineering College, \\ Salem-636 308 \\ Tamilnadu, India.
}

\begin{abstract}
Wireless local area networks (WLANs) are providing the most economical means of internet access. However, their access is very much limited. They can be deployed in a large scale by integrating them with cellular networks such as universal mobile telecommunication system (UMTS), general packet radio services (GPRS) etc. A seamless roaming between these two hetero networks can be ensured through mobile IP. But the two networks don't have support towards mobile IP which requires the deployment of home agents and a protocol between the mobile nodes, home agents and foreign agents. Hence seamless roaming can be provided by incorporating Mobile IP support in 802.11 (WLAN) networks. The cellular networks require the home agent placement to be placed and the appropriate home agents to be assigned. The home agent placement and home address assignment are the issues for supporting Mobile IP for heterogeneous roaming. If the mobiles use a home agent (HA) that is deployed in a UMTS network when roaming in a WLAN network, the UMTS network may get overloaded with WLAN traffic. The solution to this problem is to dynamically assign an HA in the WLAN domain. Different architectures can be obtained by placing HA at different positions each having its own shortcomings, in which HA is placed between border router and GGSN that requires HA performing very high speed routing as fast as GPRS gateway signaling node (GGSN) which is practically not possible occurring in the existing architecture. To overcome these difficulties different architectures are proposed in this paper. In the first architecture the HA and GGSN is connected to the border router instead of tightly coupling the HA to the GGSN, and the second architecture is HA can be collocated with one of the GGSN in UMTS network and in the third architecture, GGSN is the default router of the mobile nodes (MN) under its coverage area, the foreign agent (FA) is best collocated with the GGSN. Based on the observation of proposed architectures through performance metrics on packet overhead an attempt towards dynamic home agent assignment has been proposed.
\end{abstract}

\section{Keywords}

Home agent, high-speed routing, UMTS network, optimum network performance, mobile IP.

\section{INTRODUCTION}

Next-generation mobile/wireless all-IP networks are expected to provide a substantially wider and enhanced range of services, including global convergence, interoperability, and mobility. To support global roaming, next-generation networks will require the integration and interoperation of mobility management processes under a worldwide wireless communications infrastructure [1]. Terminal and personal mobility will enable users to access services using their personal profile, independent of terminal type and point of attachment to the network. This capability, together with the inherent IP support, is a powerful combination to deliver personalized interactive multimedia services to mobile users. A cellular data network can provide relatively low speed (up to $100 \mathrm{~kb} / \mathrm{s}$ per user) data service over a large coverage area. On the other hand, WLAN provides high-speed data service (up to $11 \mathrm{Mb} / \mathrm{s}$ with $802.11 \mathrm{~b}$ and $54 \mathrm{Mb} / \mathrm{s}$ with $802.11 \mathrm{a}$ ) over a geographically small area [2].

The interworking between WLANs and cellular networks is expected to break new ground for sophisticated business models. While $3 \mathrm{G}$ systems offer large coverage and a rich network infrastructure such as application, billing, mobility, WLANs have the potential for high data rates which allows fast Web surfing and high-quality video transmission and gaming applications. An integrated network combines the strengths of each, resulting in a wide-area system capable of providing users with ubiquitous data service ranging from low to high speed in strategic locations. Wireless local area networks based on IEEE 802.11 standard is becoming increasingly popular in providing access to the Internet [3]. WLANs are available in a wide range of devices and are now being viewed as the means of a ubiquitous broadband access platform. $802.11 \mathrm{~b}$ can provide link speeds of $11 \mathrm{Mb} / \mathrm{s}$ and application speeds of $5 \mathrm{Mb} / \mathrm{s}$, and newer standards such as $802.11 \mathrm{a} / \mathrm{g}$ provide even higher speeds, which is ideal for Internet- based data access.

Public cellular networks have evolved from the firstgeneration analog cell phones to $2 \mathrm{G}$ digital cell phone systems and $2.5 \mathrm{G}$ packet-based low-bandwidth cell phone systems to high-bandwidth all-IP wireless 3G networks; 
presently specifications are being developed for $4 \mathrm{G}$ networks. Global System for Mobile Communications is the most popular $2 \mathrm{G}$ standard; general packet radio service is its $2.5 \mathrm{G}$ and Universal Mobile Telecommunications System its 3G standard. UMTS can provide a maximum of $2 \mathrm{Mb} / \mathrm{s}$ or higher data speeds. 3G networks are designed to have wide area coverage for public access to their services; thus, they can be accessed from public areas, businesses, and hotspots. Multimedia messaging (MMS) and IP multimedia subsystem (IMS) are major services to be provided by UMTS. With IMS, users will be able to join teleconferences and receive various location-based services such as guided tours. However, it will take several years until IMS is fully available. [4].

Mobile IP is the standard protocol defined for terminal mobility in the Internet; therefore, Mobile IP is proposed as the basic mechanism to provide service continuity. Mobile IP is the key protocol to enable mobile computing and networking, which brings together two of the world's most powerful technologies, the Internet and mobile communication. In Mobile IP, two IP addresses are provided for each computer: home IP address which is fixed and care- of IP address which is changing as the computer moves. When the mobile moves to a new location, it must send its new address to an agent at home so that the agent can tunnel all communications to its new address timely. The article limits its scope to Mobile IPv4, Mobile IP for IP version 4 [5].

The Internet Protocol version 4 (IPv4) is a fundamental network layer protocol that contains addressing information and some control information that enables data packets to be routed. Mobile IPv4 can provide transparency to the upper layers while providing seamless mobility using the care-ofaddresses. It is noted that Mobile IP provides service continuity, and seamless service continuity can be provided using standardized extensions of the base protocol such as fast handover. Mobile IP requires the deployment of home agents and a protocol between the mobile nodes, home agent, and corresponding nodes. The home agent placement and home address assignment issues are addressed for supporting Mobile IP for heterogeneous roaming. The main objectives of this paper are to integrate WLAN with cellular network using different architecture and to analyze the performance of the proposed architecture.

This paper is organized as follows. The interworking between WLAN and Cellular networks scenarios is presented in Section 2. The dynamic home agent assignment is described in Section 3. Simulated results are presented in Section 4. Conclusions are given in Section 5.

\section{INTERWORKED WLAN/3G CELLULAR NETWORK}

There are two different ways to design an integrated UMTS/WLAN network architecture, defined as tight coupling and loose coupling interworking. Figure 1 shows the architecture for UMTS/WLAN integration. In a tight coupling interworking architecture, a WLAN is connected to an UMTS core network in the same manner as other UMTS radio access networks [6]. The WLAN gateway implements all the UMTS protocols required in the UMTS radio access network. In this approach, UMTS and WLAN would use the same authentication, mobility, and billing infrastructures. The main advantage of this solution is that the mechanisms for mobility, QoS, and security in the UMTS core network can be reused directly over the WLAN [7]. However, tightly coupled solutions will be highly specific to the UMTS technology and require extensive access interface standardization of WLANs beyond the existing standards. Moreover, the configuration and design of UMTS network elements, such as the serving SGSN and GGSN, have to be modified to sustain the increased traffic from WLANs. Tight coupling provides firm coupling between WLAN and GPRS, and its main advantage is enhanced mobility across the two domains, which is entirely based on GPRS mobility management protocols.

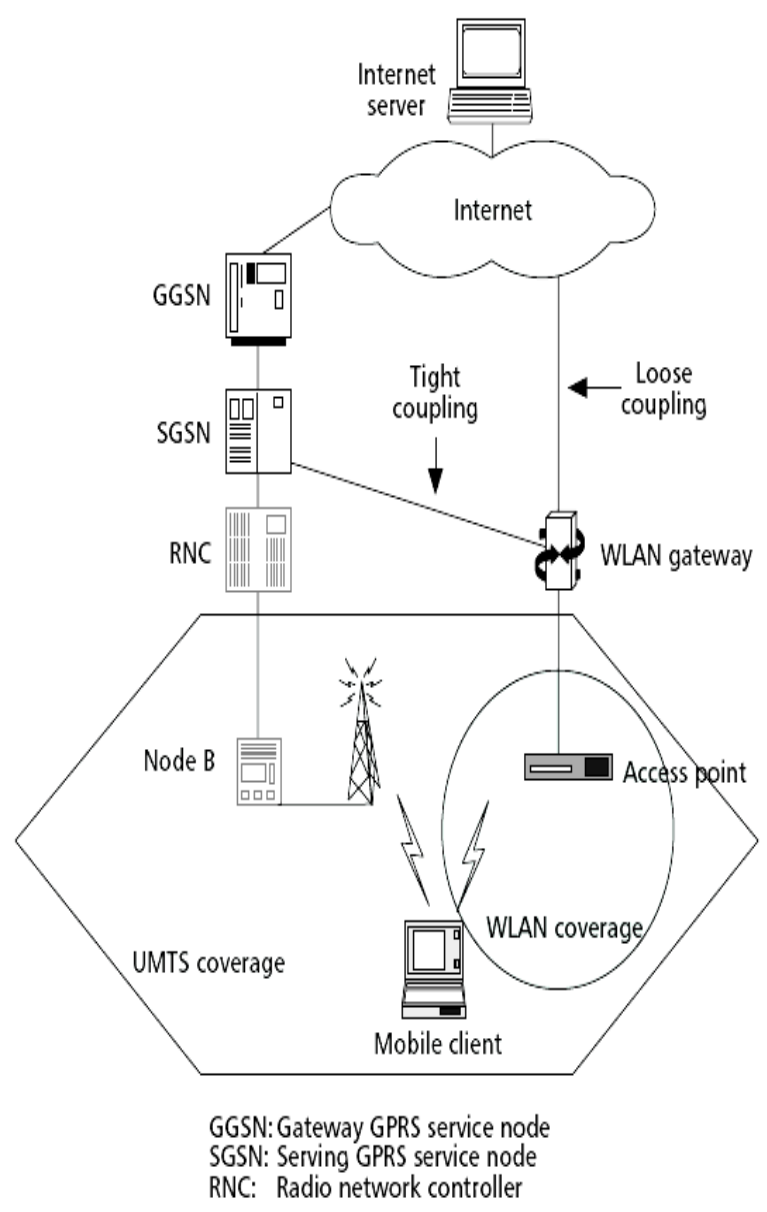

Figure 1 Integrated UMTS/WLAN systems

More important, tight coupling cannot support legacy WLAN terminals, which do not implement the GPRS protocols. These are some of the reasons that account for the current trend toward loose coupling $[8,9]$.

On the other hand, the loose interworking approach does not necessitate that the WLAN conform to the complicated 3G access/core network interfaces. Handover from WLANs to $3 \mathrm{G}$ and vice versa shall be supported. Some of the WLAN supported user services may not be supportable in $3 \mathrm{G}$ 
networks and/or may not be robust enough for the handover requirements. Services that will suffer from the latter are typically time stringent, as for the former these may be services/applications that require an amount of capacity that is not supported.. In the loose coupling approach, the WLAN gateway does not have any direct connection to UMTS network elements. WLAN traffic would not go through the UMTS core network [10]. In this approach, UMTS and WLAN can use different mechanisms and protocols to handle authentication, mobility, and billing. Nevertheless, they can share the same subscriber database for functions such as security, billing, and customer management as peer IP domains. This scheme allows the independent deployment and traffic engineering of UMTS and WLAN. Network operators and service providers can operate these two networks separately through roaming agreements. Loose coupling offers several advantages over tight coupling, such as independent deployment and traffic engineering of UMTS and WLANs.

\section{DYNAMIC HOME AGENT ASSIGNMENT}

There are several requirements for HA placement. For users of UMTS, the HA should be placed in a UMTS network. However, UMTS did not incorporate Mobile IP into its architecture, so any new entities to be added must have minimal impact on overall UMTS operations. Mobile IP requires the deployment of the home agents and a protocol between the mobile nodes, home agent, and the corresponding nodes. In WLANs IP mobility can only be handled with Mobile IP. For seamless communication of MNs roaming between the two networks, Mobile IP needs to be supported in both GPRS/UMTS and WLAN networks. When an HA is placed in a UMTS network for mobiles that roam in WLANs, traffic from CNs is routed to the UMTS network. Assuming the $\mathrm{CN}$ will send on average at the application rate of $5 \mathrm{Mb} / \mathrm{s}$, the traffic increase in and out of UMTS may reach 1 Gbps if the number of mobiles in WLANs exceeds 200. This fact also places another requirement: trying to minimize the effects of traffic increase. Four different architectures are presented with which to place an HA in a UMTS network.

\subsection{Existing Architecture}

An HA can be placed between the border router and a specific GGSN. The GGSN needs to be selected by all mobiles that need to use Mobile IP during the PDP context establishment phase in UMTS access. The mobile is always connected to the home network when it is in UMTS, so it does not need any registration with its HA.

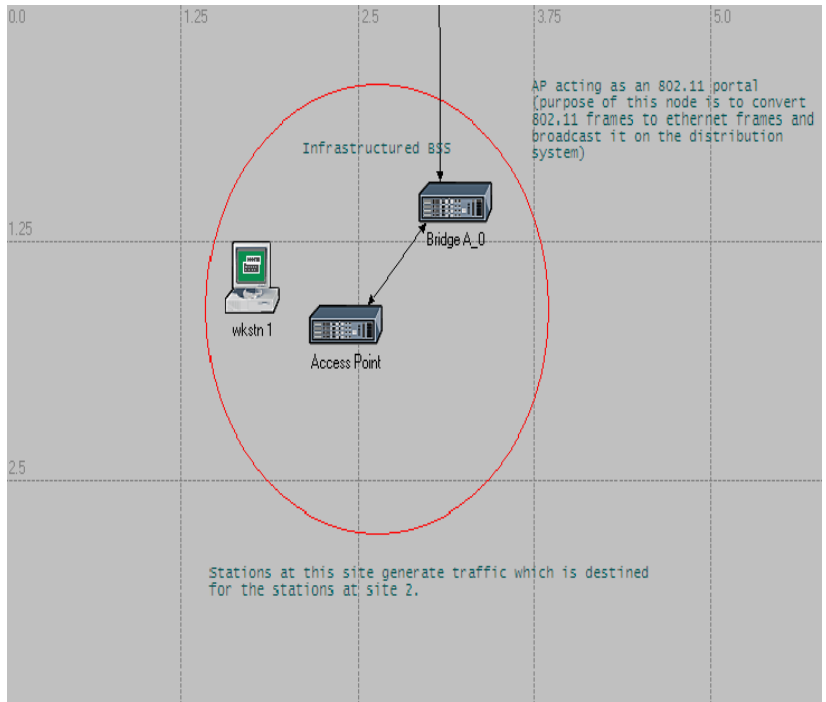

Figure 2 Subnet model of MN in WLAN

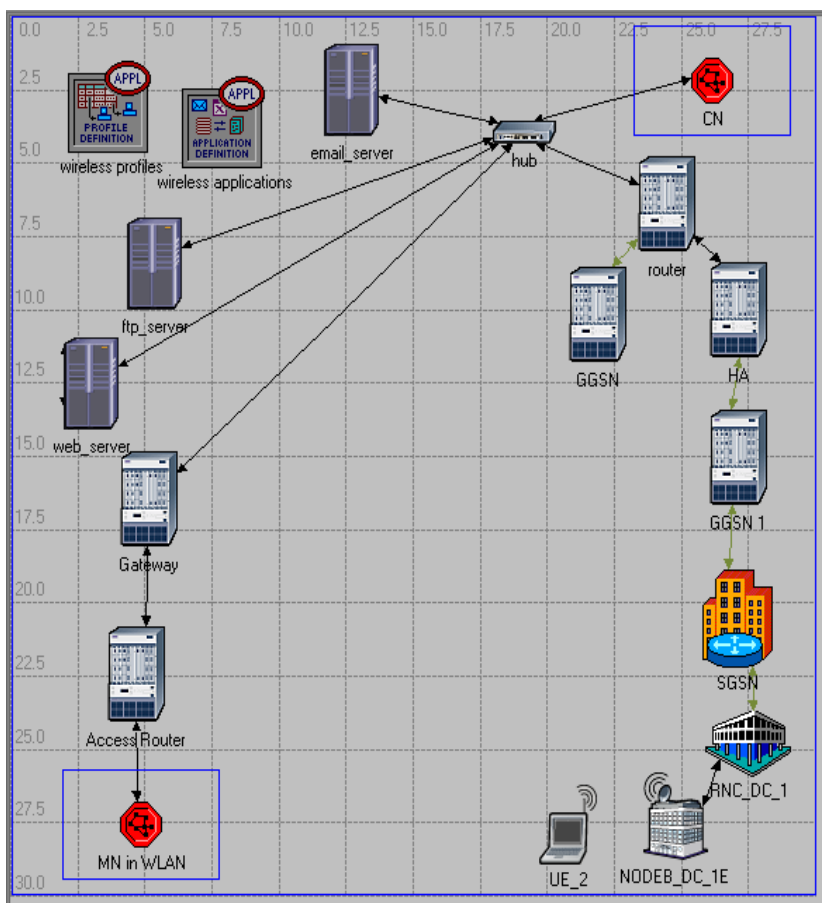

Figure 3 Existing Architecture

An FA in the WLAN domain can be placed at an AR, as shown in the Figure 3; if no FA is deployed, the MN has to use collocated CoA mode. In UMTS, due to the placement of the HA, if an MN establishes PDP context with the specific GGSN, the MN, from the Mobile IP point of view, is considered at home, so no Mobile IP support is needed. Even if the MN roams and changes SGSNs, it only needs to use link layer mobility provided by GTP tunnels. The MN in WLAN and CN shown in architectures is a subnet which is comprised of OPNET attributes as shown in figure 2 


\subsection{Proposed Architecture 1}

An HA can be collocated with one of the GGSNs in a UMTS network as shown in Figure 5 The MNs that need Mobile IP services select this GGSN during PDP context establishment. The address assigned to the PDP context is also used as the mobile's HoA. In a UMTS network, as in existing architecture, MNs are in their home network and therefore do not need any Mobile IP services. Collocating the GGSN with the HA works well except when there is a clash in behavior: the GGSN terminates any active PDP contexts if the $\mathrm{MN}$ is roaming in WLAN hotspots and is not in the UMTS, and the address is released. The HA, however, has to keep this address in order to perform tunneling for the MN.

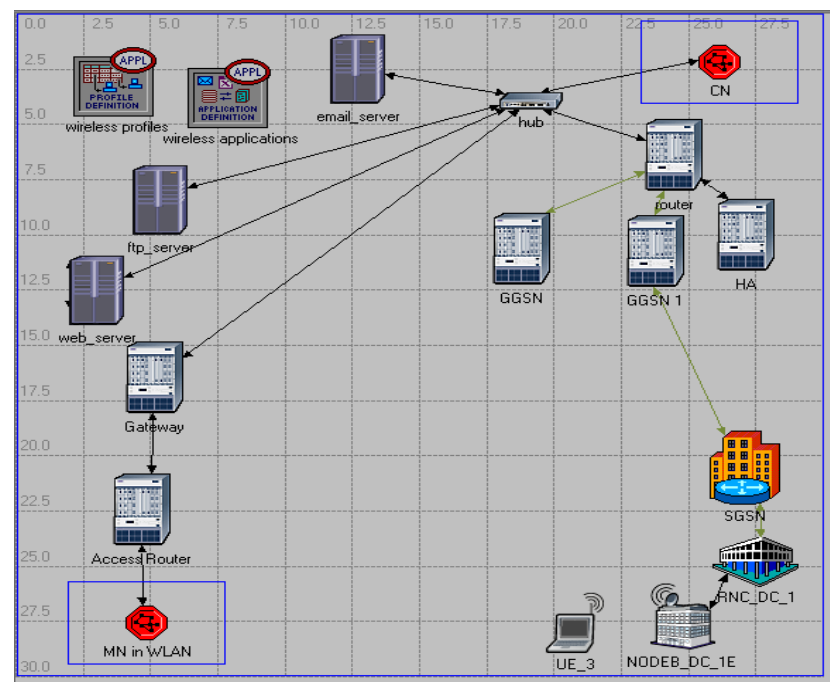

Figure 4 Proposed architecture 1

\subsection{Proposed Architecture 2}

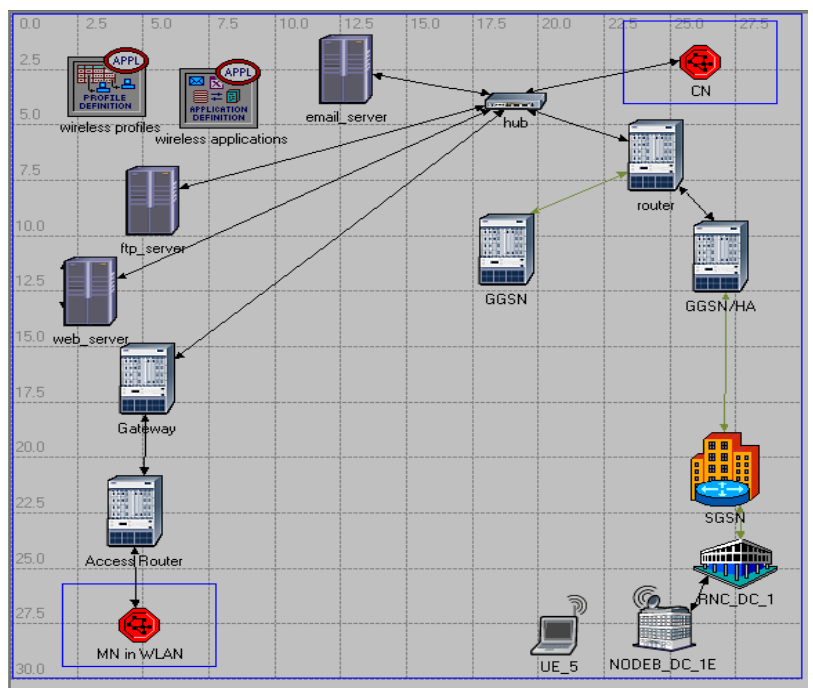

Figure 5 Proposed architecture 2

\subsection{Proposed Architecture 3}

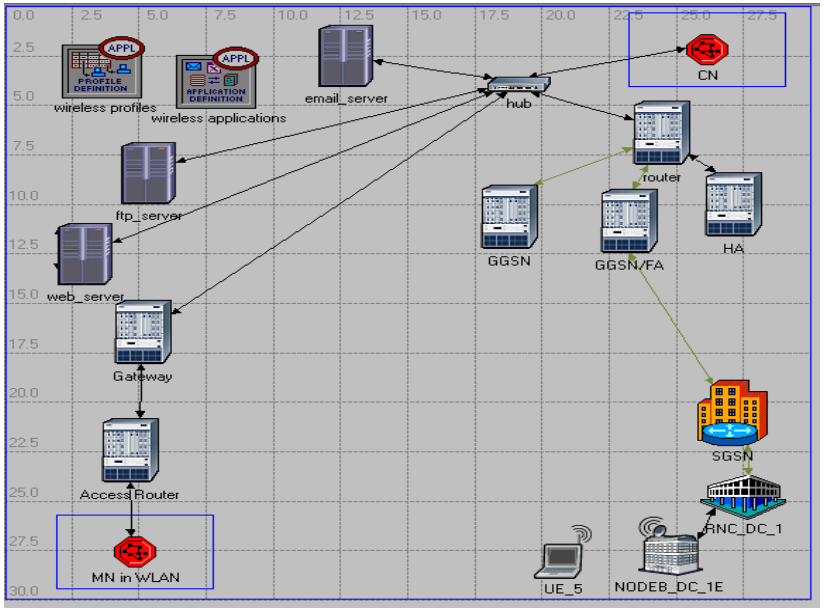

Figure 6 Proposed architecture 3

As shown in Figure 6, architecture 3 is a slight modification of proposed architecture 1 with the addition of an FA. Since the GGSN is the default router of the MNs under its coverage area, the FA is best collocated with the GGSN. In this architecture the MN, when roaming in UMTS, needs to select a different GGSN and use it as an FA in getting a CoA (the address associated with the PDP context), and register this address with its HA

\section{SIMULATION RESULTS}

The main objective is to integrate WLAN with cellular networks using different architecture by providing NAT/NAPT traversal the performance are evaluated and the performance analysis such as latency and packet overhead of this architecture are simulated using optimum network performance (OPNET) and analysed. The placement of HA in the architecture and routing it in different position provides a difference in their performance. In UMTS, Constant bit rate (CBR), Variable bit rate (VBR), - Available bit rate (ABR),UBR - Unspecified bit rate (UBR) are used for queue configuration.

\subsection{Performance of Existing Architecture}

In the existing architecture, an HA is placed between the border router and a specific GGSN. The Figure 7 depicts maximum traffic sent through the WLAN is approximately $1 \mathrm{Mbps}$ and the traffic received is nearly $1.75 \mathrm{Mbps}$ which is a converged network of UMTS and WLAN. This slow access is due to the traffic which has to pass through HA in between the border router and a specific GGSN. In UMTS network, the Figure 8 infers that $12 \mathrm{kbps}$ is the transmit load and the received load is $9 \mathrm{kbps}$. Advantages of architecture this are its simplicity, intrinsic Mobile IP support, and minimal change to UMTS architecture.

A disadvantage of this architecture is that an HA must be able to perform very high-speed routing, as fast as a GGSN. Advantages of existing architecture are its simplicity, intrinsic Mobile IP support, and minimal change to UMTS 
architecture. A disadvantage of this architecture is that an HA must be able to perform very high-speed routing, as fast as a GGSN

\subsection{Performance of Proposed Architecture 1}

The HA and GGSN can be connected to the border router instead of tightly coupling the HA to the GGSN. An HA in this architecture now can serve all GGSNs in the domain. The Figure 9 explains the maximum traffic sent through the WLAN is approximately $1 \mathrm{Mbps}$ and the traffic received is nearly 2.54 Mbps. This fast access is due to the HA and GGSN connected to the border router and a specific GGSN. In UMTS network, the Figure shown in 10 infer that $42 \mathrm{kbps}$ is the transmit load and the received load is $40 \mathrm{kbps}$.

The advantages of this architecture are its simplicity and scalability. Only one new entity is added to the UMTS network, and the solution is scalable because the HA can be scaled as the number of Mobile IP users increase. Compared with existing architecture, an HA does not need to route all GGSN traffic, which is another advantage for this architecture

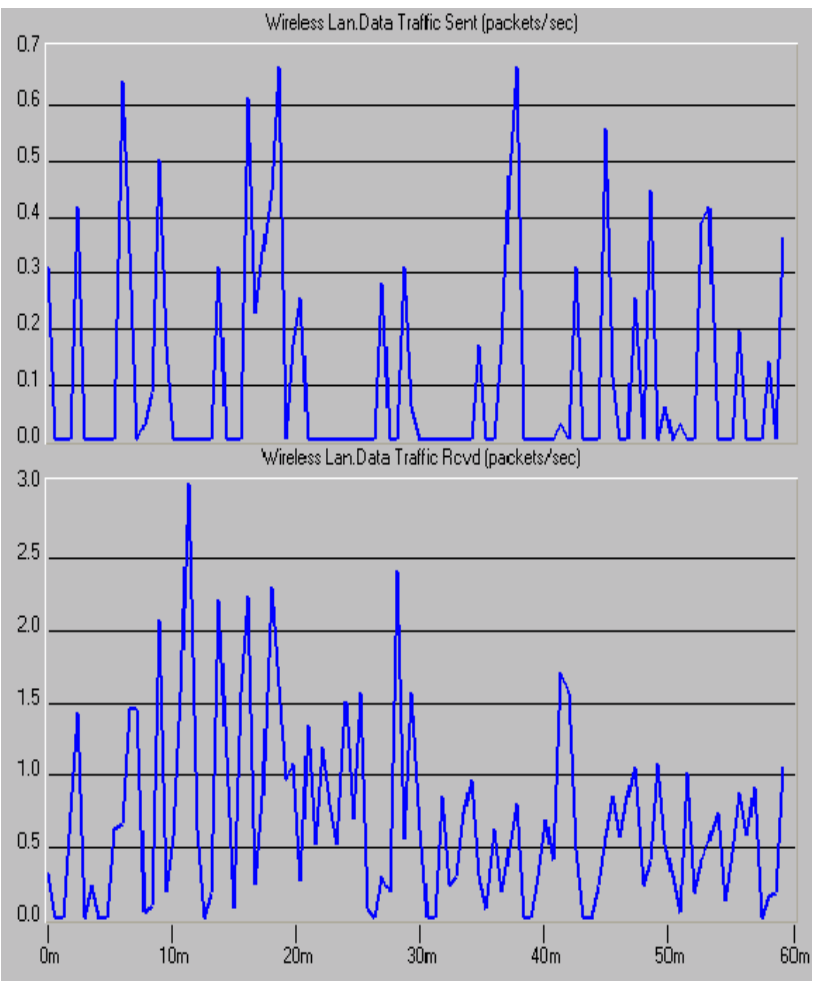

Figure 7 Performance of WLAN in existing architecture

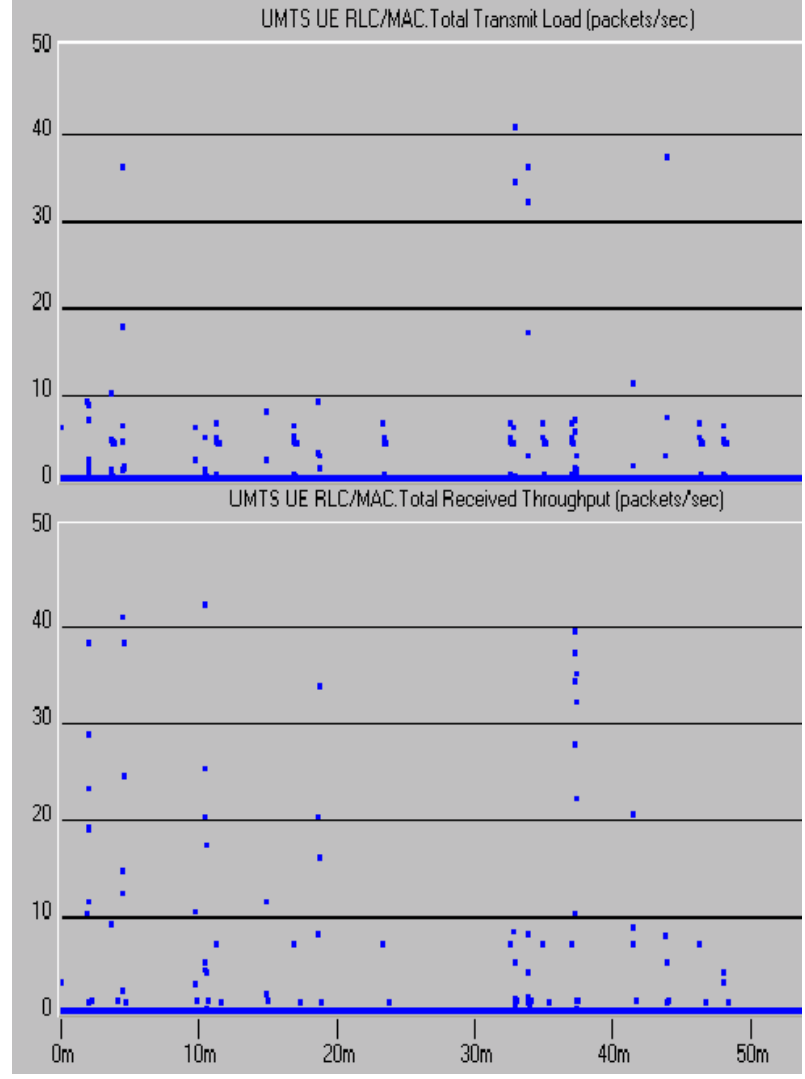

Figure 8 Performance of UMTS in existing architecture

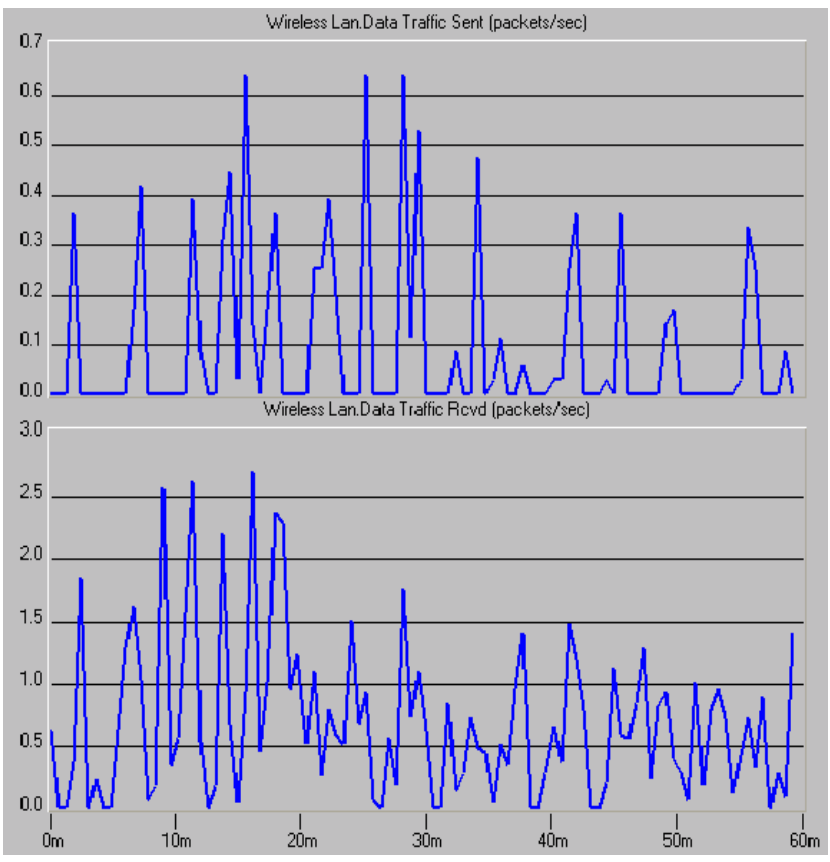

Figure 9 Performance of UMTS in proposed architecture 


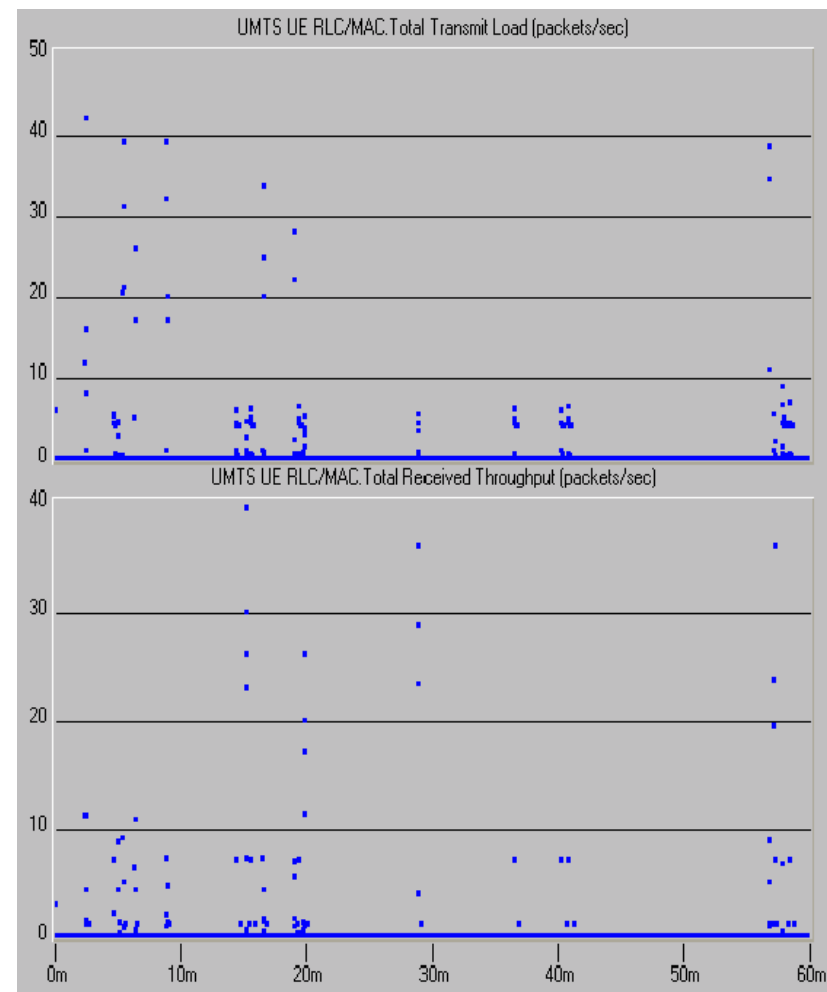

Figure 10 Performance of UMTS in proposed architecture 1

\subsection{Performance of Proposed Architecture 2}

This architecture is a slight modification of existing architecture. The HA is collocated with one of the GGSNs in cellular network. The Figure 11 depicts maximum traffic sent through the WLAN is approximately $1 \mathrm{Mbps}$ and the traffic received is nearly $2.84 \mathrm{Mbps}$. This fast access is due to the HA is collocated with one of the GGSNs. In UMTS network, the Figure 12 infers that $41 \mathrm{kbps}$ is the transmit load and the received load is the same.

An advantage of an HA being collocated with a GGSN is that in this case the HA does not need to perform proxy address resolution protocol (ARP) interactions for the MNs. In a UMTS network, as in architecture 1, MNs are in their home network and therefore do not need any Mobile IP services and this adds another advantage to cellular network.

The disadvantages of architecture 2 are the load of HA operations that are added to the GGSN and the need to reserve the HoAs assigned to the MNs when they roam into WLANs. These disadvantages do not exist in existing architecture. Among the proposed architectures this architecture founds to be better because of its better result in WLAN which is a converged series and its optimal routing in UMTS network.

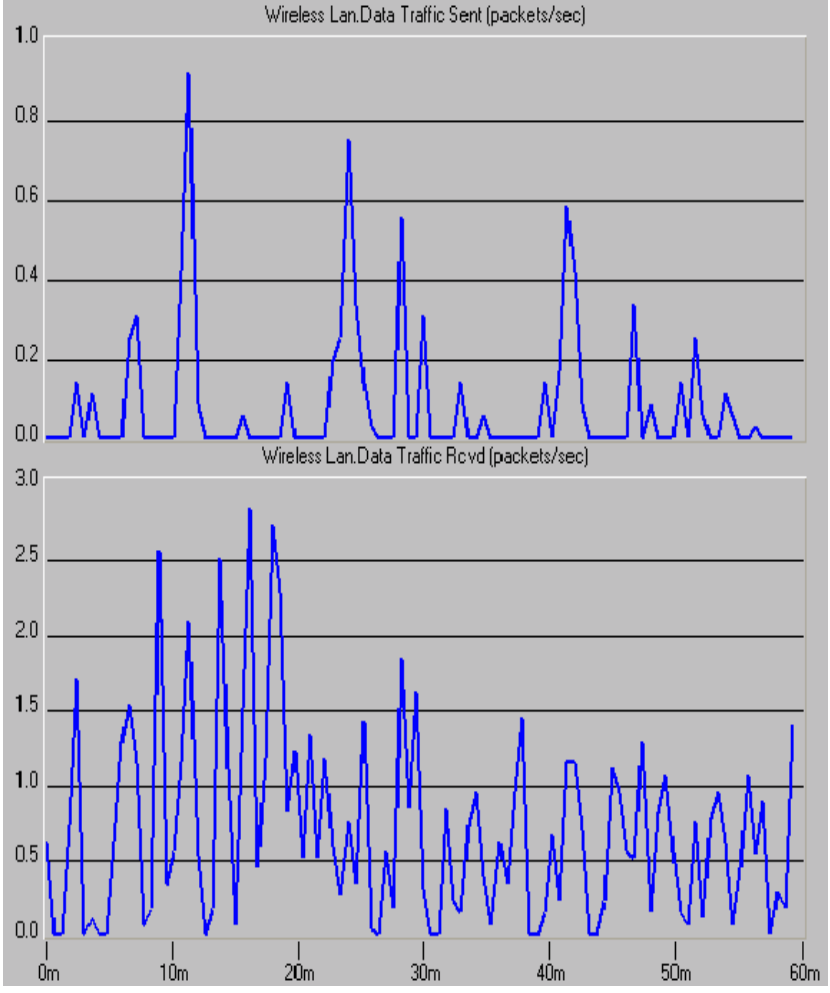

Figure 11 Performance of WLAN in proposed architecture 2

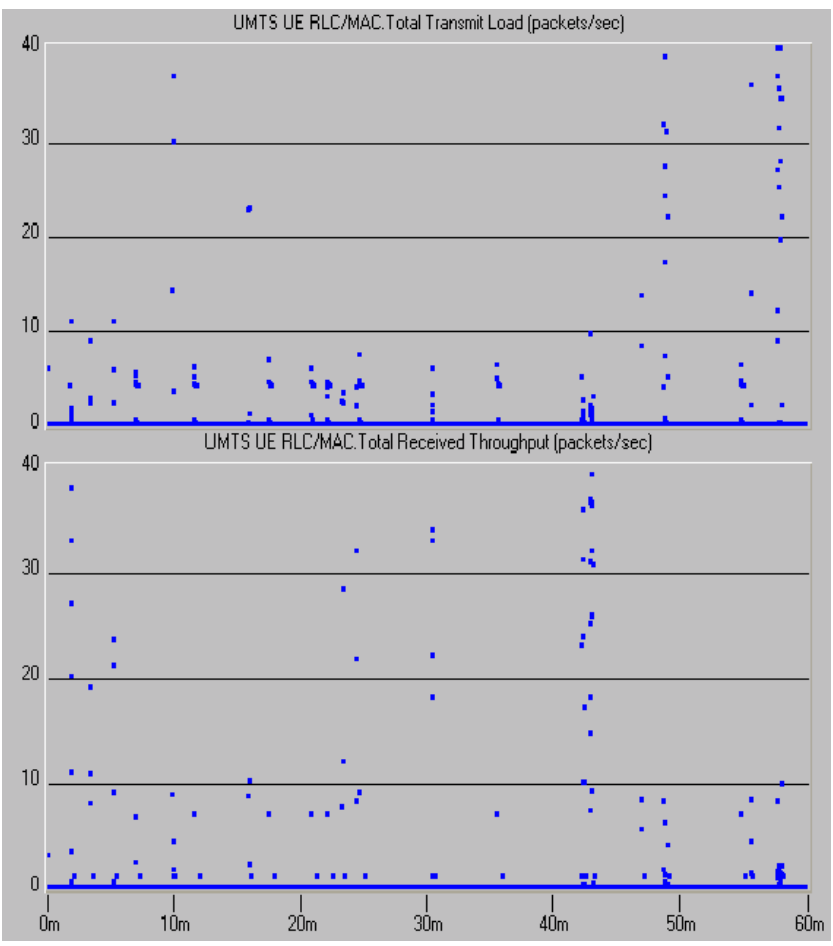

Figure 12 Performance of UMTS in proposed architecture 2 


\subsection{Performance of Proposed Architecture 3}

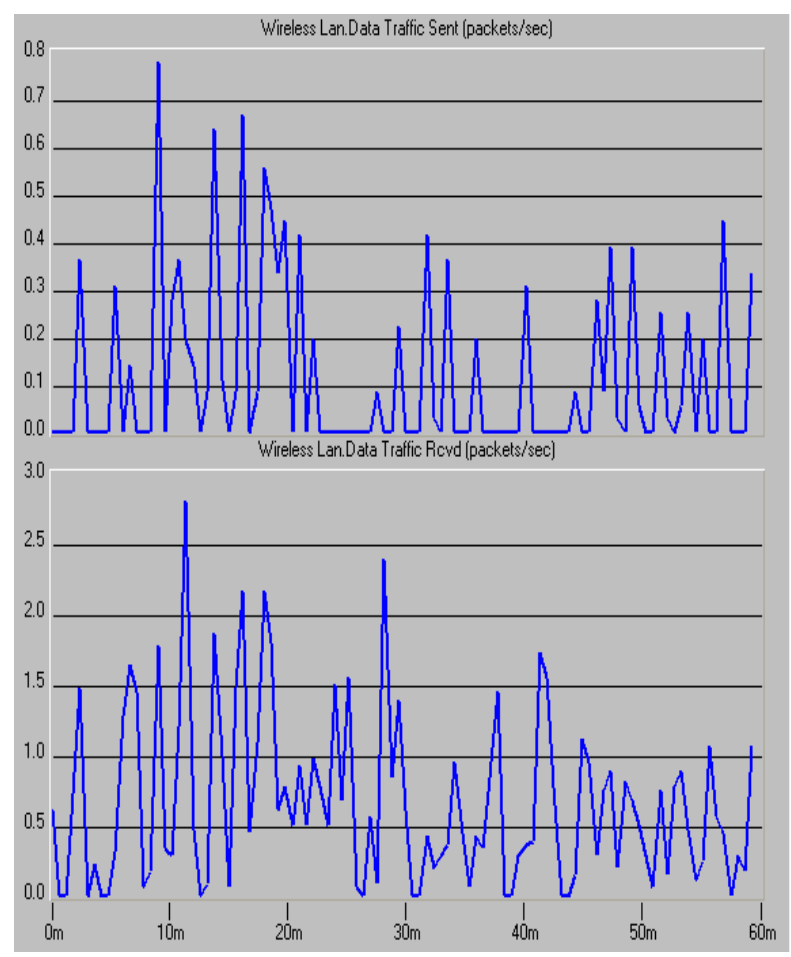

Figure 13 Performance of WLAN in proposed architecture 3

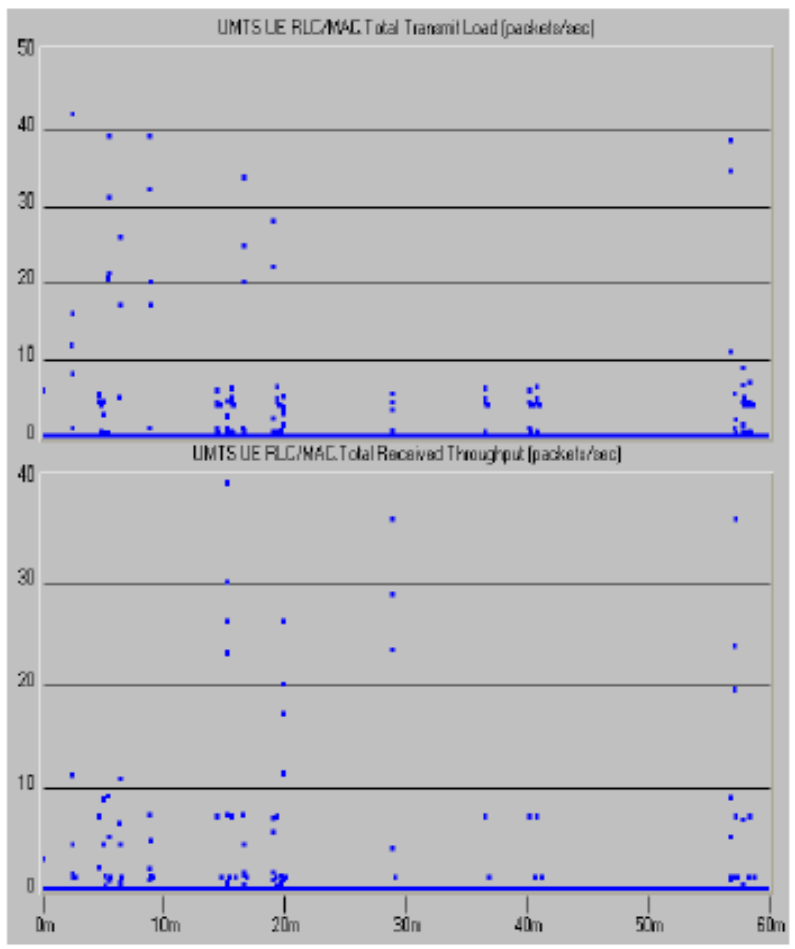

Figure14 Performance of UMTS in proposed architecture3
Architecture 3 is a slight modification of architecture 1 with the addition of an FA. Here in the WLAN as shown in Figure 13 the traffic sent is $1 \mathrm{Mbps}$ and received about $2.75 \mathrm{Mbps}$ where as in UMTS network, the Figure 14 infers that 41 kbps is the transmit load and the received load is $40 \mathrm{kbps}$. Since the GGSN is the default router of the MNs under its coverage area, the FA is best collocated with the GGSN. In architecture 3 the MN, when roaming in UMTS, needs to select a different GGSN and use it as an FA in getting a CoA (the address associated with the PDP context), and register this address with its HA.

The disadvantage of this architecture is that the operation of Mobile IP is a partial replacement of UMTS' GTP; GTP and Mobile IP together introduce redundant mobility handling operations such as encapsulation/decapsulation in the data path.

\section{CONCLUSION}

Various architectures that can be used in practice, each has its own advantage. HA placement plays an inferior role in delay, but during data transfer, the closer the HA is placed, the higher the bandwidth. Simplicity, intrinsic mobile IP support and minimal change to UMTS architecture are advantages and HA must be able to perform very high-speed routing, as fast as a GGSN which is the disadvantage of existing architecture. Advantages of proposed architecture are its simplicity and scalability.

Compared with existing architecture, an HA does not need routes all GGSN traffic, which is another advantage for these architectures. An advantage of an HA being collocated with a GGSN is that in this case the HA does not need to perform proxy address resolution protocol (ARP) interactions for the MN. There are still several challenges like mobility management, Qos, unified billing to be addressed to enable seamless interworking of WLAN and UMTS networks

\section{ACKNOWLEDGMENTS}

Our thanks to ACM for allowing us to modify templates they had developed.

\section{REFERENCES}

[1] Ian F. Akyildiz, Janise McNair, Joseph Ho, Huseyin Uzuinalioglu, and Wenye Wang "Mobility anagement in Current and Future Communication Networks", IEEE Network, vol. 12, no. 8, pp.39-49, July 2005.

[2] K. Salkintzis, "Interworking Techniques and Architectures for WLAN/3G Integration Towards 4G Mobile Data Networks," IEEE Wireless Commun., vol. 11, no. 3, June 2004, pp. 50-61.

[3] P. S. Henry and H. Luo, "Wi-Fi: What's next?", IEEE 
Communication Magazine, vol. 40, no. 2, pp. 66-72, December 2002.

[4] J. Luo, R. Mukerjee, M. Dillinger, E. Mohyeldin, and E. Schulz, "Investigation of Radio Resource Scheduling in WLANs coupled with 3G Cellular Network", IEEE Communication Magazine, vol. 41, no. 6, pp. 108-115, June 2003.

[5] C. E. Perkins, "Mobility Support in IPv4", Internet Engineering Task Force, RFC 3344, August 2002.

[6] Wei Song, Hai Jiang. and Weihua Zhuang, "Performance Analysis of the WLAN-First Scheme in Cellular/WLAN Interworking", IEEE Wireless Communication, Vol. 6, No. 5, May 2007.

[7] Kalle Ahmavaara, Henry Haverinen, and Roman Pichna,"Interworking Architecture Between 3GPP and WLAN Systems," IEEE Wireless Communications, vol. 9, no. 5, pp. 112-24. November 2002.
[8] Li Ma, Fei Yu, And Victor C. M. Leung, "A New Method to Support UMTS/WLAN Vertical Handover Using SCTP", IEEE Wireless Communication, vol. 11, no.4, pp. 44-51, August 2004.

[9] M. Buddhikot et al., "Integration of 802.11 and ThirdGeneration Wireless Data Networks," IEEE INFOCOM 2003, April 2003.

[10] Dimitrios I. Axiotis, Tareq Al-Gizawi, "Services in Interworking $3 \mathrm{G}$ and WLAN Environments", IEEE Transactions on Communication,vol.47, no. 12, pp. 1897-1904, December 2004. 